Reprod. Nutr. Dévelop., 1988, 28 (1), 187-188.

\title{
Variations inter-individuelles d'insulinémie et de quelques paramètres du métabolisme énergétique chez la vache en fin de $2^{e}$ mois de lactation
}

\author{
P. FAVERDIN
}

Station de Recherches sur la Vache Laitière, I.N.R.A., St-Gilles, 35590 L'Hermitage, France.

Summary. At the end of the 2 nd month of lactation, the insulinemia of dairy cows before the meal was poorly correlated with milk production level, and there was no relation with the deficit status of the animal. The increase of insulinemia during the meal was mainly related to glycemia.

Une liaison négative entre l'inșulinémie et le niveau de production laitière a été observée par Hart et al. (1978) et Walsh et al. (1980); cependant elle pourrait n'être due qu'à un effet du stade physiologique de lactation puisqu'elle n'a pas été significative entre des animaux au même stade (Koprowski et Tucker, 1973). Cet essai avait pour but $d^{\prime}$ 'étudier en fin de $2^{\mathrm{e}}$ mois de lactation la relation interindividuelle entre l'insulinémie et la production ou la qualité du lait et des paramètres du bilan énergétique. L'insulinémie a été caractérisée soit par sa valeur basale (avant le repas du matin), soit par sa variation au cours du repas.

Matériel et méthodes. Une population de 26 vaches pie-noires multipares a été répartie suivant les résultats de la lactation précédente en 4 groupes en croisant les facteurs niveaux de production laitière et taux moyens de matières grasses (4 500 à 6500 et 6500 à $8500 \mathrm{~kg}$ de lait, 33 à 43 et 43 à $53 \mathrm{~g}$ de matières grasses par $\mathrm{kg}$ de lait).

A la $8^{e}$ semaine de lactation, 3 prélèvements sanguins ont été effectués sur chaque vache dans la veine de la queue avant la distribution du repas, 90 et 120 min après. Les animaux recevaient une ration composée d'ensilage de maïs distribué à volonté et d'aliments concentrés apportés proportionnellement à la production de lait attendue. Les aliments étaient retirés $12 \mathrm{~h}$ avant le premier prélèvement de sang. L'hématocrite, l'insuline, le glucose et les acides gras non estérifiés (AGNE) ont été dosés dans le plasma, selon les méthodes standard (Faverdin, 1985). Les corrélations ont été calculées suivant la méthode des rangs de Spearman (rs) et les lots ont été comparés à l'aide du test $U$ de Mann Whitney en raison de l'absence de normalité de certaines variables.

Résultats et discussion. L'insulinémie basale varie de 5 à $23 \mu \mathrm{U} / \mathrm{ml}$ suivant les animaux, mais la différence entre vaches à moyen et haut potentiel de production est peu importante (tabl. 1). La corrélation entre insulinémie basale et production laitière, bien que significative ( $r s=-0,35, P<0,05$ ) est très faible, et I'ACP (fig. 1) montre que les populations des moyennes et des fortes productrices sont assez mal séparées suivant la direction de l'insulinémie basale. Par ailleurs l'insulinémie basale ne présente aucune liaison avec la glycémie et l'état de mobilisation des réserves lors des prélèvements (AGNE et bilan énergétique) ou pendant les semaines précédentes (variations de poids vif). L'insulinémie et les autres constituants sanguins n'ont pas été reliés de manière significative aux différences inter-individuelles de teneur du lait en matières grasses. 
L'augmentation de l'insulinémie consécutive à la prise du repas est corrélée positivement avec la glycémie avant repas ( $r s=0,61, P<0,001$ ) et avec la quantité de matière sèche ingérée ( $r s=0,35, P<0,05)$. Elle est indépendante de la production laitière et de l'insulinémie basale. La diminution de la glycémie pendant le même temps est reliée à la variation de l'insulinémie au cours du repas ( $r s=-0,45, P<0,01)$, ce qui est bien représenté par la position diamétralement opposée de ces 2 variables dans l'ACP.

En conclusion, entre vaches laitières à la même semaine de lactation, la $8^{e}$, les différences d'insulinémie, avant le repas ou en réponse à celui-ci, ne peuvent être clairement reliées ni aux différences de production et de composition du lait, ni au bilan énergétique. Par ailleurs, l'insulinémie et la glycémie présentent des relations entre individus similaires à celles observées intra-individus (Faverdin, 1985).

TABL. 1. - Effet du potentiel de production des vaches sur les teneurs plasmatiques en insuline, glucose et acides gras non estérifiés (AGNE) avant repas ( $\mathrm{t} 0)$ et sur leur variation pendant $(\mathrm{t} 120$ - $\mathrm{t} 0$ )

\begin{tabular}{cccccccc}
\hline $\begin{array}{c}\text { Potentiel } \\
\text { laitier }\end{array}$ & $\begin{array}{c}\text { Insuline } \\
\mathrm{t} 0\end{array}$ & $\begin{array}{c}\mu \mathrm{U} / \mathrm{mi} \\
\mathrm{t} 120-\mathrm{t} 0\end{array}$ & $\begin{array}{c}\text { Glucose } \\
\mathrm{t} 0\end{array}$ & $\begin{array}{c}\mathrm{mg} / \mathrm{di} \\
\mathrm{t} 120-\mathrm{t} 0\end{array}$ & $\begin{array}{c}\text { AGNE } \mu \mathrm{mole} / \mathrm{ml} \\
\mathrm{t0}\end{array}$ & Bilan UFL \\
\hline$<6500 \mathrm{~kg}$ & 11,5 & 12,6 & 58 & -8 & 240 & -189 & $-1,8$ \\
$>6500 \mathrm{~kg}$ & 8,7 & 11,2 & 53 & -6 & 396 & -330 & $-3,4$ \\
Seuil test $\mathrm{U}$ & $\mathrm{P}<0,05$ & $\mathrm{P}>0,1$ & $\mathrm{P}<0,01$ & $\mathrm{P}>0,1$ & $\mathrm{P}<0,01$ & $\mathrm{P}<0,01$ & $\mathrm{P}<0,1$ \\
\hline
\end{tabular}

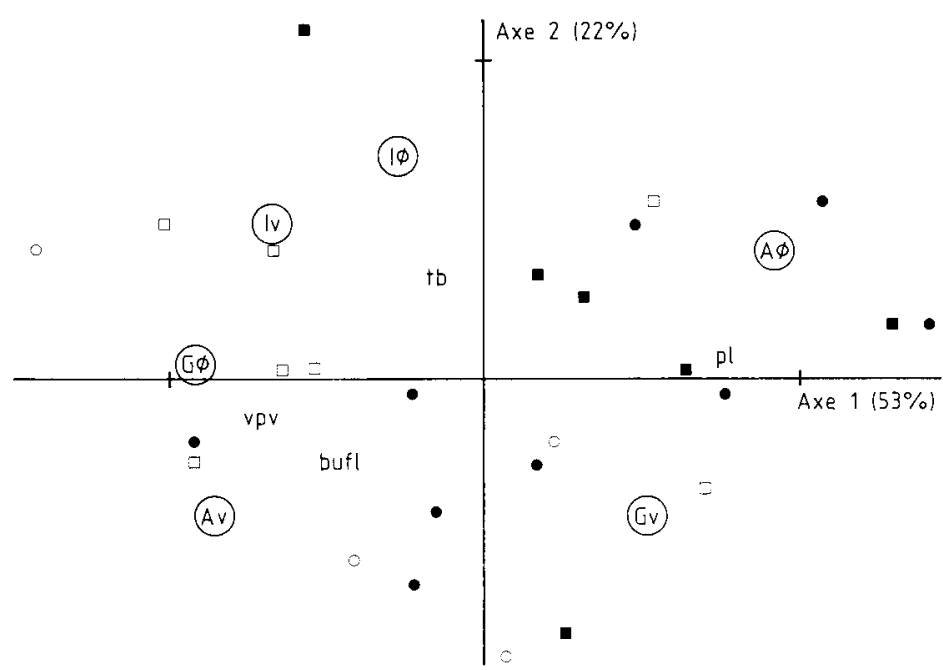

FIG. 1. - Analyse en composantes principales de quelques paramètres plasmatiques chez des vaches en $8^{e}$ semaine de lactation. La dispersion de la population a été étudiée sur les teneurs en insuline, glucose et acides gras non estérifiés avant le repas (respectivement $\mathrm{IO}, \mathrm{GO}, \mathrm{AO}$ ) ainsi que sur les variations de ces paramètres en réponse au repas (respectivement Iv, Gv, Av). Les variables production laitière (pl), taux butyreux (tb), variations de poids vif (vpv) et bilan énergétique (bufl) ont été introduites en variables supplémentaires. Caractéristiques individuelles au cours de la lactation antérieure : () $\mathrm{pl}<6500$ et tb $<43$, $\square$ pl $<6500$ et tb $>43$, - pl $>6500$ et tb $<43, \square$ pl $>6500$ et tb $>43$.

Faverdin P., 1985. Thèse de Docteur-Ingénieur de I’INAPG, 133 p.

Hart I. C., Bines J. A., Morant S. V., Ridley J. L., 1978, J. Endocr., 77, 333-345.

Koprowski J. A., Tucker H. A., 1973. Endocrinology, 93, 645-651.

Walsh D. S., Vesely J. A., Mahadevan S., 1980. J. Dairy Sci., 63, 290-294. 